\title{
The Study of Body Image, Self-esteem and Sexual Satisfaction of College Students in Southern Taiwan
}

\author{
Hsiu-Chou Lin, Yen-Chin Lin*
}

Graduate School of Human Sexuality, Shu-Te University, Kaohsiung 82445, Taiwan

Copyright $\mathrm{O} 2018$ by authors, all rights reserved. Authors agree that this article remains permanently open access under the terms of the Creative Commons Attribution License 4.0 International License

\begin{abstract}
The purpose of the study is to assess the relationship between sexual satisfaction and gender, perception of body image, and level of self-esteem in college students in southern Taiwan. This study conducted questionnaires completed by 637 college students in southern Taiwan. The questionnaire consisted of four sections: the Personal Background Survey, the Multidimensional Body-Self Relations Questionnaire, the Rosenberg Self-esteem Scale, and the Sexual Satisfaction Inventory. Statistical analysis used in the study included descriptive statistical, t-test, Pearson correlation, and stepwise multiple regression. Major findings of this study are as follows: 1. In perception of body image, level of self-esteem and sexual satisfaction are between medium and high in college students in southern Taiwan. 2. Evaluation of appearance and body satisfaction in male was significantly higher than female; orientation of appearance in female was significantly higher than male and sexual satisfaction in male was significantly higher than female. There was no significant difference between male and female in self-esteem. 3 . The positive relationships were found to be significant in college students in southern Taiwan between perception of body image and self-esteem, perception of body image and sexual satisfaction, and self-esteem and sexual satisfaction. 4. Gender, perception of body image, and self-esteem were the main predictors for sexual satisfaction in college students in southern Taiwan. The researcher sincerely hopes that this study can provide the consulters in colleges some ideas in regards to students' body image, self-esteem, and sexual satisfaction, which in turn can help them resolve students' sex problems in the future.
\end{abstract}

Keywords Body Image, Self-esteem, Sexual Satisfaction, College Students in Southern Taiwan

\section{Introduction}

Human beings are born with sexual interest, sexual drive, sexual desire, and multidimensional response to sex. Human's feeling and thinking, learning and language, and many other resources achieve human biological responses and accumulate the life experience [1]. Therefore, sex includes the physical body and psychological personality. People agree that sex is an integral part of human being's entire lifetime. Sex is not just for reproduction, but also plays an important role in daily life. Sexual satisfaction is the reaction of individual emotion. This response comes from the assessment of the positive and negative subjectivity of the individual in the sexual relationship, which includes: the satisfied degree of the sexual attitude, sexual relationship and sexual desire [2]. Individuals who have a healthy and satisfied development in sex will be more confident and happier in life and are more equipped to deal with the challenges of life and have a brighter future.

"Sex" involves a major process of development for college students who are in the late adolescences. It is also important to build a sense of security and healthy interpersonal relationships. As Taiwan, in East Asia, is bound by the traditional moral of the East, as well as under the impact of the open social culture from the West, Taiwanese college students have become inquisitive and confused about sex. Therefore, under the drive of sexual self-exploration and engagement, occurrence of premarital sexual behaviors of college students is increasing. Sex, which is human natural, is a part of love. On that account, a more tolerant attitude is needed for the premarital sexual behaviors of college students.

In early Taiwanese society, "sex" was regarded as a taboo and obscenity, and sexual behavior was examined under strict moral principles. Accordingly, premarital sexual behavior was considered as a prohibited behavior [3]. Moreover, there were double standards for sexual behavior between men and women in Taiwan's society. The concept of "chastity" was mainly imposed to women, and women's premarital sexual behaviors were generally regarded as a cultural stigma [4]. Therefore, Taiwanese women, being compared with men, adopted a more conservative attitude and behavior toward premarital sex under the code of "chastity". However, in recent years, because of social and cultural changes in Taiwan, along with independence and gender awareness of women, the gender inequality of the 
traditional concept of "chastity" has gradually been challenged [5]. As a result, to cope with a phenomenon of increasing Taiwanese younger sexual behavior which is derived from the changing attitude about sex, it is vital to call a special attention to sexual education of young Taiwanese.

According to Erikson's stages of psychosocial development, the main task in adolescence is self-identity. If adolescents' psychological, emotional, and social identities develop smoothly, they may be able to identify their roles better culturally and sexually. But if the self-identity does not develop smoothly, they will experience the role of confusion, low self-control and low sense of security. Through the process of self-identification, quite a few adolescents tend to focus on their appearance, in the goal of building a better self-image and to impress others. Body image is the part of self-concept and the sum of individuals' attitudes and experiences [6]. It is an accommodation and dynamic process that is continuously interacting with society [7]. Therefore, body image is also one of the multidimensional factors in sex. According to the Social Comparison Theory, the individual's satisfaction of body image is the result of comparison with others. Individuals tend to have a natural drive to force themselves to compare with either society's standards or social celebrities [8]. Hence one naturally falls into the process of "comparing upwards". As a result, the individual's emotional pressure is increased and self-esteem is reduced. When the body image is low, it will lead to a lower sexual self-esteem (personal perception of their sex) psychologically and emotionally. Being shy about the body will lead to lower sexual satisfaction of the sexual experience in intimate relationships [9]. Moreover, dissatisfaction with appearance and excessive psychological anxiety on body appearance will lead him/her to sexual relationships that make him/ her feel shy and try to avoid physical exposure. That will also reduce sexual desire, sexual enjoyment and sexual performance [10]. Either distortion or depression on the body image and the low self-esteem can lead to the inability to enjoy healthy and satisfying relationships [1].
Counseling centers in the university campus often help students to explain and deal with physiological and psychological doubts. These counseling centers also allow students to seek counseling for interpersonal relationships, some examples are: how to get along with the opposite sex, how to maintain and deal with conflict in an intimate relationship, and even the interpersonal relationship about sex. The issue of sex on campus nowadays is more widespread than ever. More and more college students are seeking counseling about sex. It is thus essential to examine how college students' perception of body image to influence their level of self-esteem and intimate behaviors.

The purpose of the study is to assess the relationship between sexual satisfaction and gender, perception of body image, and level of self-esteem in college students in southern Taiwan. The major purposes of this study are as follows:

Realize the level of perception of body image, self-esteem, and sexual satisfaction of the college students in southern Taiwan.

Examine the difference in the level of perception of body image, self-esteem, and sexual satisfaction between male and female college students.

Analyze the relationship among the level of perception of body image, self-esteem, and sexual satisfaction.

Predict the level of sexual satisfaction based on gender, perception of body image, and self-esteem of the college students in southern Taiwan.

\section{Methods}

The purpose of the study is to understand the relationship between sexual satisfaction and gender, perception of body image, and level of self-esteem in college students in southern Taiwan. The framework of this study is described in Figure 1.

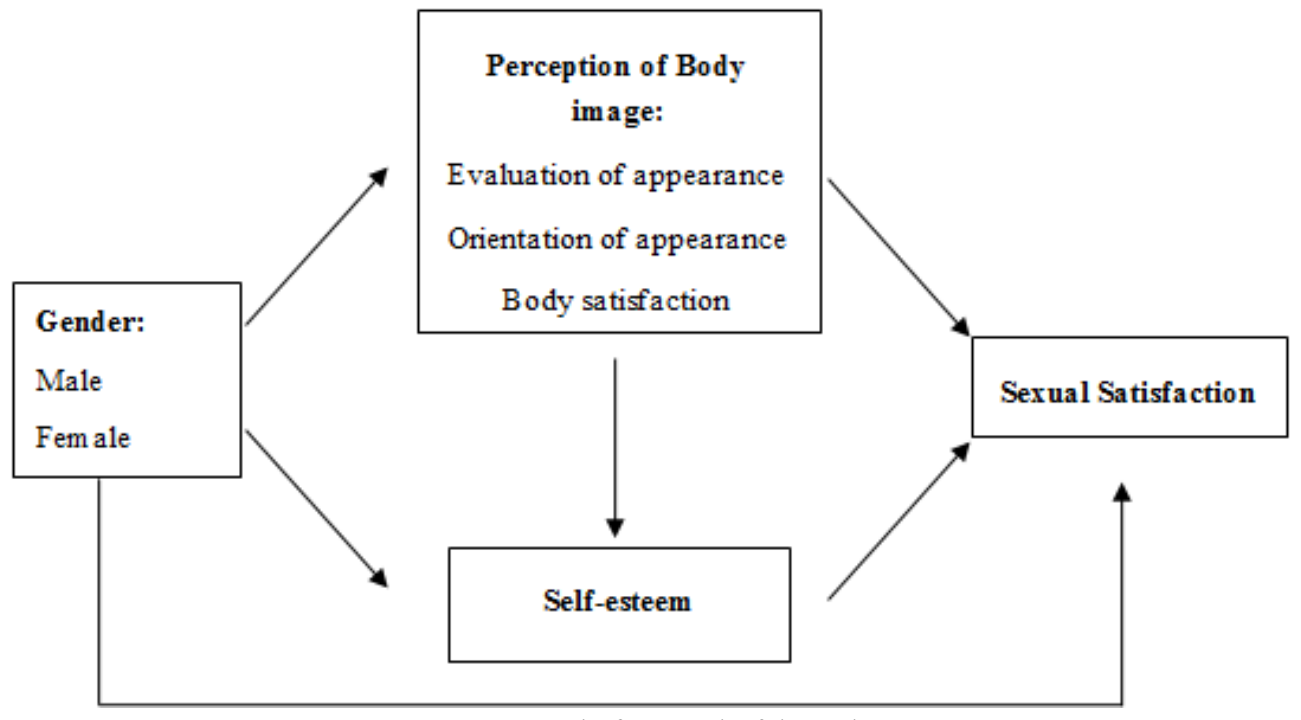

Figure 1. The framework of the study 
This study conducted questionnaires completed by 637 college students in southern Taiwan. The questionnaire consisted of four sections: the Personal Background Survey, the Multidimensional Body-Self Relations Questionnaire, the Rosenberg Self-Esteem Scale (SES), and the Sexual Satisfaction Inventory. Statistical analysis used in the study included descriptive statistical, t-test, Pearson correlation, and stepwise multiple regression.

This study used the cross-sectional survey with descriptive and inferential statistics method. The population of the study is the college students in the southern Taiwan area including Kaohsiung city, Tainan city and Pingtung city. Because of the high autonomy of college students and high privacy issue about sexual satisfaction, this study took the purposive sampling method. The time of this study began from April 1 to April 20. 702 students who completed questionnaire serve as the sample for this study. The effective questionnaires were 637; the efficiency of the questionnaire is $91 \%$.

The structured questionnaire consisted of four sections: the Personal Background Survey, the Multidimensional Body-Self Relations Questionnaire, the Rosenberg Self-Esteem Scale, and the Sexual Satisfaction Inventory. All of them used Likert's six-point scale for scoring. Among the questionnaires:

Body-Self Relations Questionnaire referenced by Yen-Yen $\mathrm{Yu}$ [11] was translated from Cash's multidimensional body and self-relationship scale, total of 27 items;

The Rosenberg Self-Esteem Scale (SES), total of 10 items;

Sexual Satisfaction Inventory (SSI) [1] developed by Whitley in 1975 and modified by the researcher to be the Satisfaction Scale for Taiwan college students, total of 18 items.

The validity and the reliability of the questionnaire included face validity and content validity. The Cronbach's $\alpha$ coefficient was used to verify the consistency of the Multidimensional Body-Self Relations Questionnaire, the Rosenberg Self-Esteem Scale, and the Sexual Satisfaction Inventory. For the first 40 college students who did the pre-test, the Cronbach's $\alpha$ was between 0.7 and 0.9 (Table1) which was in the range of acceptable consistency within the questionnaire [12].

Table 1. The Cronbach's $\alpha$ of questionnaire

\begin{tabular}{ccc}
\hline $\begin{array}{c}\text { The Name of } \\
\text { Questionnaire }\end{array}$ & $\begin{array}{c}\text { Cronbach's } \alpha \\
\text { (pre-test=39) }\end{array}$ & $\begin{array}{c}\text { Cronbach's } \alpha \\
\text { (test=637) }\end{array}$ \\
\hline $\begin{array}{c}\text { Body-Self Relations } \\
\text { Questionnaire } \\
\text { Rosenberg Self-Esteem } \\
\begin{array}{c}\text { Scale } \\
\text { Sexual Satisfaction } \\
\text { Inventory }\end{array}\end{array}$ & .88 & .85 \\
\hline
\end{tabular}

\section{Conclusions}

There were 637 college students, including 261 male students $(41.0 \%)$ and 376 female students (59.0\%). Major findings of this study are as follows:

\subsection{The Level of Perception of Body Image, Self-esteem, and Sexual Satisfaction}

3.1.1. The level of perception of body image: the average of the perception of body image is 3.95 , which is higher than the median (3.5). It is between medium and high in college students in southern Taiwan. Evaluation of the appearance and body satisfaction in male is significantly higher than in female; orientation of appearance in female was significantly higher than in male (Table 2).

3.1.2. The level of self-esteem: the average of the self-esteem is 4.13 , which is higher than the median (3.5). It is between the medium and high range of college students in southern Taiwan. And there was no significant difference between male and female (Table 2).

3.1.3. The level of sexual satisfaction: the average of the sexual satisfaction is 4.47 , which is higher than the median (3.5). It is between the medium and high range in college students in southern Taiwan. The results also imply that sexual satisfaction in male is significantly higher than in female (Table 2).

Table 2. The level of perception of body image, self-esteem and sexual satisfaction $(\mathrm{N}=637)$

\begin{tabular}{cccccccc}
\hline & \multicolumn{3}{c}{ Total } & \multicolumn{7}{c}{ Male(n=261) } & \multicolumn{2}{c}{ Female(n=376) } & \multirow{2}{*}{$\mathrm{t}$} \\
\cline { 2 - 7 } & $\mathbf{M}$ & SD & $\mathbf{M}$ & SD & M & SD & \\
\hline Perception of body image & 3.95 & .56 & 3.99 & .61 & 3.92 & .52 & 1.49 \\
Evaluation of appearance & 3.62 & .78 & 3.72 & .83 & 3.55 & .75 & $2.671 * *$ \\
Orientation of appearance & 4.53 & .71 & 4.36 & .74 & 4.64 & .67 & $-5.13 * * *$ \\
Body satisfaction & 3.70 & .80 & 3.89 & .85 & 3.57 & .73 & $5.11^{* * *}$ \\
Self-esteem & 4.13 & .65 & 4.10 & .71 & 4.14 & .60 & -.70 \\
Sexual satisfaction & 4.47 & .86 & 4.72 & .82 & 4.28 & .84 & $6.64 * * *$ \\
\hline$* * p<.01 ; * * * p<.001$ & & & & & & &
\end{tabular}


Table 3. The relationship among the level of perception of body image, self-esteem, and sexual satisfaction (N=637)

\begin{tabular}{cccc}
\hline Independent variable & Perception of body image & Self-esteem & Sexual satisfaction \\
\hline Perception of body image & & & \\
Self-esteem & $.537^{* *}$ & \\
Sexual satisfaction & $.378^{* *}$ & $.367^{* *}$ & \\
\hline
\end{tabular}

${ }^{* *} p<.01 ; * * * p<.001$

\subsection{The Correlation among the Level of Perception of Body Image, Self-esteem, and Sexual Satisfaction}

3.2.1. The significantly high and positive relationships were found in college students in southern Taiwan between perception of body image and self-esteem $(\mathrm{r}=.537, \mathrm{p}<.01)$. It showed that the more positive perception of body image, the higher the self-esteem (Table 3).

3.2.2. The significantly medium and positive relationships were found in college students in southern Taiwan between perception of body image and sexual satisfaction $(\mathrm{r}=.378, \mathrm{p}<.01)$. It showed that the more positive perception of body image, the higher the sexual satisfaction (Table 3 ).

3.2.3. The significantly medium and positive relationships were found in college students in southern Taiwan between self-esteem and sexual satisfaction $(\mathrm{r}=.367$, $p<.01)$. It showed that the more positive self-esteem, the higher the sexual satisfaction (Table 3 ).

\subsection{Gender, Perception of Body Image, and Self-esteem were the Main Predictors for Sexual Satisfaction}

The best prediction is the perception of body image, $14.3 \%$, followed by gender, $5.4 \%$, and self-esteem, $4.4 \%$. The equation of stepwise multiple regressions for the results is:

$$
\begin{aligned}
& \text { Sexual satisfaction }=1.963-.434 \times \text { Gender }+.351 \times \\
& \text { Perception of body image }+.333 \times \text { Self-esteem. }
\end{aligned}
$$

\subsection{Suggestions from the Study}

\subsubsection{Purge the Culture of the Media Today and Enhance the} Autonomy of the Body Image

It is found that an individual's satisfaction of body image is influenced by social culture. Appearance is like a social stimulus, easily lead others to set expectations and make assumption on it. Expectations and assumption reflect on the "ideal figure" that the society and culture created. The mass media plays a vital role in advocating the image of "an ideal figure"- thin with curve, no flaws and a unified body image in society. The more Individuals are exposed to media messages, the easier they shape their own body image to their favorite media role [13], which in turn leads adolescents' unrealistic attitudes and displeasure toward their body image. Student's time in college is the later stage of adolescence, which is a very important stage in pursuit of self-identification. During this stage of rapid physical and psychological development, their ability to self-develop, to self-establish, and to self-identify is very important. Acceptance of body image constitutes the root of adolescent adaptation and plays an important factor in psychological anxiety.

The researcher suggests college students are encouraged to accept their body image and not to be manipulated by the mass media, which tends to materialize female in our society. Moreover, college students should learn to appreciate the multivariate beauty, affirm and praise their own body-looking and appearance. In addition, it is vital to rebuild an index for a realistic and healthy figure. Proper adjustment of the subjective feelings and beliefs about the body image will increase the satisfaction of body image and lead to a healthy and positive perception of body image [14]. As teachers, discuss with college students about the "ideal body" that the mass media tries to deliver to our society as a common fashion and provide clarification of value. That will help students have a reasonable and practical expectation toward their body image. Moreover, it is believed that the higher satisfaction of body image, the higher satisfaction of their intimate relationships, with regard to the degree of satisfaction with self-identity and self-confidence [15].

\subsubsection{Advocate Facing Nudity, Educate to Respect Human Body}

The results of this study show that the satisfaction and acceptance of one's self naked is not high, which means in the oriental culture, sex is given multiple and profound meanings. It is both beautiful and evil, and both can be praised and can be tabooed.

College students should learn to appreciate the beauty of nudity and to think positively about sex. Traditionally, sex is always regarded as obscenity and become a taboo in the oriental society. However, it is important that college students have to identify and decline inappropriate messages and attitudes that promote incorrect, shame, or fear about the images of nudity and sex. College students should be taught to respect the nudity and enjoy the excitement from seeing naked body and touching during sex, which in turn will improve their sexual satisfaction. By inspiring the students' aesthetic instincts, we can help them respect themselves and others' bodies. This represents a social progress and openness, and students have multiple 
value orientation of the diversity in the freedom of life.

\subsubsection{Advocate Sexual Education to Enhance Sexual Satisfaction}

Sex in human society evolves from a simple concept to the practice of interpersonal relationships. In general, sex is the pleasure of physical contact, but also the way of love craving, but also involves the relationship between marriage and reproduction. Sex also involves identity of gender roles, power of relationship, physical aesthetics, as well as physical function. Therefore, sex is the most basic and complex problems of life [16].

In fact, adolescents are in the process of seeking for self-identity and intimate relationship. College students in their later period of adolescents are naturally attracted to sexual behaviors to some degree. Advocates of the more inclusive approaches argue that some students will have sex no matter what they are told, and they need to learn how to protect themselves. Sexual education programs teach college students to identify correct and incorrect information about sex, to have the ability discussing ethical and social issues about sex, and to understand that safe sex and pleasurable sex are compatible. Sexual education programs might be more well-proceeded by the way of re-conceptualizing safe sex in pleasurable terms. This is because if we can make safe sex more desirable to students, they may be more inclined to practice it [17]. For this end, the counselor and teacher may teach students to pursue pleasurable safer sex by using techniques such as condom, educate students by how to have better sex, and assist students by how to improve their dialogue about sex [18]. Therefore, it is necessary to realize college students' attitudes toward sex and offer them information about sex and related legal issues. College students are provided with a wide range of information and the focus is developing decision-making skills when it comes to sexual activity [17]. Moreover, the idea that responsibility goes along with joy should be implanted so both parties may take precautions before having sex.

Nowadays, more and more college students visit counseling centers in campus with the counselor to inquire about sexual information and to discuss the unsatisfying sexual relationships that has caused one of their interpersonal problems. Thus, to best address the sexual health needs of college students, school-based sexual education must go beyond talking only about abstinence and the dangers of sexual activity [17]. While the knowledge of safe sex is emphasized, it is also vital to understand that sexual desire and sexual pleasure are both indispensable elements of sex. The students, teachers or counselors not only need open-minded and realistic attitudes towards sex but also require capabilities to talk sexual issues in a multivariate and comfortable way. Therefore, the teachers need to have good knowledge of human sexuality to help with these problems. The researcher sincerely hopes that this study can provide the counselors and teachers in colleges to have some concepts regarding to students' body image, self-esteem, and sexual satisfaction, which in turn can help them to resolve students' sexual problems in the future.

\section{Acknowledgements}

First of all, thanks to my family, in my study and writing process continued to encourage and support me. Moreover, thanks to Dr. Yen-Chin Lin, the director of Graduate School of Human Sexuality of Shu-Te University provided me more guidance during the study. Thanks to 702 college students who are willing to participate the study, so that this study can proceed smoothly. Thank you very much for every person who guides, helps, encourages and supports me during my study and writing.

\section{REFERENCES}

[1] R. Walker-Hill. An Analysis of the Relationship of Human Sexuality Knowledge, Self-esteem, and Body Image to Sexual Satisfaction in College and University Students (Unpublished Doctoral Dissertation), The University of Tennessee, USA, 1998.

[2] K. Lawrane, E. S. Byerrs. Sexual satisfaction in heterosexual long-term relationship: The interpersonal exchange model of sexual satisfaction, Personal Relationships, Vol.2, No.2, 267-285, 1995

[3] H. W. Yen. Sex, Sexual Relationships and Sexual Education, Psychological Publishing, Taiwan, 2004.

[4] C. G. Lee. The evolvement of the history of "Chastity" and its modern enlightenment, Journal of Confucius-Mencius Society of the Republic of China, Vol.75, 187-202, 1998.

[5] H. W. Yen, C. H. Liu, C. C. Cheng. Trends of dating and sex behavior among fifth grade students of Junior colleges in Taipei city from 1979 to 2007, Taiwan Guidance and Counseling Association, Vol.25, 251-274, 2009.

[6] M. E. Drench. Changes in body image secondary to disease and injury, Rehabilitation Nursing, Vol.19, No.1, 31-36, 1994.

[7] Y. Y. Hsu, C. H. Chen. Analysis of body concept, Nursing Journal, Vol.46, No.6, 103-109, 1999.

[8] L. J. Heinberg, J. K. Thompson. The effect of figure size feedback and target comparison group on body image, International Journal of Eating Disorder, Vol.12, 441-448, 1992.

[9] J. S. Leopold. The Direct and Indirect Effect of Body Image on Sexual Satisfaction (Unpublished Doctoral Dissertation), ST. Mary's University of San Antonio, USA, 2002.

[10] T. F. Cash, C. L. Maikkula, Y. Yamamiya. Baring the body in the bedroom: Body image, sexual self-schemas, and sexual functioning among college women and men, Electronic 
Journal of Human Sexuality, Vol.7, 2004.

[11] Y. Y. Yu. The Study of Somatotype and Body Image Related Factors among the Students in Chung Yuan Christian University (Unpublished Doctoral Dissertation), National Taiwan Normal University, Taiwan, 2001.

[12] S. K. Lo. The Reliability and Validity of Research Tools. In S. Lee (Eds.), Nursing Research: Principles and Practice, Farseeing Press, Taiwan, 2003.

[13] K. Eyal, Y. Te'eni-Harari. Explaining the relationship between media exposure and early adolescents' body image perceptions, Journal of Media Psychology, Vol.25, 129-141, 2013.

[14] K. J. Thompson, L. J. Heinberg, M. N. Altabe, S. Tantleff-Dunn. Exacting Beauty: Theory, Assessment, and
Treatment of Body Image Disturbance, American Psychology Association, USA, 1999.

[15] H. L. Wei. The study on Adults' Body Image and Relationship Satisfaction in Greater Taipei Area (Unpublished Master's Thesis), Chinese Culture University, Taiwan, 2016.

[16] Y. C. Li. Sex and life, The Journal of Gender Equity Education, Vol.14, 117-119, 2001.

[17] J. J. Lehmiller. The Psychology of Human Sexuality, John Wiley \& Sons Ltd, UK, 2014.

[18] A. Philpott, W. Knerr, V. Boydell. Pleasure and prevention: when good sex is safer sex, Reproductive Health Matters, Vol.14, 23-31, 2006. 